Nonl. Analysis and Differential Equations, Vol. 1, 2013, no. 1, 15 - 22 HIKARI Ltd, www.m-hikari.com

\title{
Mean Square Exponential Stability for Stochastic Functional Differential Equations with Impulses
}

\author{
Nan Ding \\ School of Mathematics and Statistics \\ Chongqing Three Gorges University \\ Wanzhou 404100, China \\ dingnan8118@126.com
}

Copyright (c) 2013 Nan Ding. This is an open access article distributed under the Creative Commons Attribution License, which permits unrestricted use, distribution, and reproduction in any medium, provided the original work is properly cited.

\begin{abstract}
In this paper, stochastic functional differential equations with impulses are considered. By employing Gronwall-Bellman inequality, the stochastic analytic technique and the properties of operator semigroup, the sufficient conditions ensuring the exponential stability in mean square for mild solution of such system are obtained. Our results can generalize and improve the existing works.
\end{abstract}

Mathematics Subject Classification: 34K45, 60H15, 93E15

Keywords: Mean square exponential stability, Stochastic, Impulse

\section{Introduction}

Stochastic partial differential equations have attracted the attention of many authors and many valuable results on the stability of the solution have been established, see $[4,5,6,9,11,13]$ and references therein. For example, Taniguchi [13] has considered the exponential stability for stochastic partial differential equations by the energy inequality; Caraballo and Liu [9] have investigated the exponential stability for mild solution to stochastic partial differential equations with delays by utilizing the Gronwall inequality; Liu and Shi [6] have considered the exponential stability for stochastic partial functional differential equations by means of the Razuminkhin-type theorem; Taniguchi [11] has 
proved the almost sure exponential stability of mild solution for stochastic partial functional differential equation by using the analytic technique; Luo [4-5] has discussed asymptotic stability of stochastic partial differential equations with infinite delays and exponential stability for mild solutions of stochastic partial differential equation with delays by fixed point theorem, respectively.

On the other hand, impulsive effects likewise exist in a wide variety of evolutionary processes, for example, medicine and biology, economics, mechanics, electronics and telecommunications, etc., in which many sudden and abrupt changes occur instantaneously, in the form of impulses. Many interesting results on impulsive effects have been obtained, see $[1,3]$ and references therein. When we consider the exponential stability for mild solutions of stochastic partial functional differential equations with impulses, the main difficulty mainly comes from impulsive effects on the system since the corresponding theory for such problem has not yet been fully developed. Many excellent tools to derive the exponential stability for mild solution of stochastic partial functional differential equations may be difficult and even ineffective for the exponential stability of such system with impulses. Therefore, there is few results on the stability for stochastic partial functional differential equations with impulses, see [7-8] and references therein.

Motivated by the above discussion, in this paper, by using Gronwall-Bellman inequality, the stochastic analytic techniques and the properties of operator semigroup, we obtain some new sufficient conditions to ensure the exponential stability in mean square for mild solution of stochastic partial functional differential equations with impulses. Our results can generalize and improve the existing works.

The rest of this paper is organized as follows. In section 2, we present some basic notations and definitions. In section 3, sufficient conditions are derived to ensure the exponential stability in mean square for mild solution.

\section{Preliminary Notes}

Let $H, K$ be two real separable Hilbert spaces and $\mathcal{L}(K, H)$ be the space of bounded linear operators mapping $K$ into $H$. For convenience, we shall use the same notations $\|\cdot\|$ to denote the norms in $H, K$ and $\mathcal{L}(K, H)$ without any confusion. Let $\left(\Omega, \mathrm{F},\{\mathrm{F}\}_{t \geq 0}, \mathbb{P}\right)$ be a complete probability space with a filtration $\{\mathrm{F}\}_{t \geq 0}$ satisfying the usual conditions (i.e., it is right continuous and $\mathrm{F}_{0}$ contains all $\mathbb{P}$-null sets). Let $\{\omega(t): t \geq 0\}$ denote a $K$-valued $\{\mathrm{F}\}_{t \geq 0^{-}}$ Wiener process defined on $\left(\Omega, \mathrm{F},\{\mathrm{F}\}_{t \geq 0}, \mathbb{P}\right)$ with covariance operator $Q$, i.e.,

$$
\mathrm{E}<\omega(t), x>_{K}<\omega(s), y>_{K}=(t \wedge s)<Q x, y>_{K}, \quad x, y \in K,
$$

where $Q$ is a positive self-adjoint, trace class operator on $K,<\cdot, \cdot>_{K}$ denotes the inner product of $K, \mathrm{E}$ denotes the mathematical expectation. In particular, 
we call such $\omega(t): t \geq 0$, a $K$-valued $Q$-Wiener process with respect to $\{F\}_{t \geq 0}$.

In order to define stochastic integrals with respect to the $Q$-Wiener process $\omega(t)$, we introduce the subspace $K_{0}=Q^{1 / 2}(K)$ of $K$ which, endowed with the inner product $\left\langle u, v>_{K_{0}}=<Q^{-1 / 2} u, Q^{-1 / 2} v>_{K}\right.$ is a Hilbert space. We assume that there exists a complete orthonormal system $\left\{e_{i}\right\}_{i \geq 1}$ in $K$, a bounded sequence of nonnegative real numbers $\lambda_{i}$ such that $Q e_{i}=\lambda_{i} e_{i}, i=1,2 \cdots$, and a sequence $\left\{\beta_{i}(t)\right\}_{i \geq 1}$ of independent Brownian motions such that

$$
<\omega(t), e>=\sum_{i=1}^{\infty} \sqrt{\lambda_{i}}<e_{i}, e>\beta_{i}(t), \quad e \in K
$$

and $B_{t}=B_{t}^{\omega}$, where $B_{t}^{\omega}$ is the sigma algebra generated by $\{\omega(s): 0 \leq s \leq t\}$. Let $\mathcal{L}_{2}^{0}=\mathcal{L}_{2}\left(K_{0}, H\right)$ denote the space of all Hilbert-Schmidt operators from $K_{0}$ into $H$. It turns out to be a separable Hilbert space, equipped with the norm

$$
\|\psi\|_{\mathcal{L}_{0}^{2}}^{2}=\operatorname{tr}\left(\left(\psi Q^{1 / 2}\right)\left(\psi Q^{1 / 2}\right)^{*}\right) \text { for any } \psi \in \mathcal{L}_{2}^{0} .
$$

Clearly, for any bounded operators $\psi \in \mathcal{L}(K, H)$, this norm reduces to $\|\psi\|_{\mathcal{L}_{2}^{0}}^{2}=$ $\operatorname{tr}\left(\psi Q \psi^{*}\right)$.

Let $\mathbb{R}$ and $\mathbb{Z}$ be the sets of real and integer numbers, respectively; $\mathbb{R}^{+}=$ $[0,+\infty)$ and $C(X, Y)$ denotes the space of continuous mapping from the topological space $X$ to the topological space $Y$. Especially, $C \triangleq C([-\tau, 0], \mathbb{R})$ denotes the family of all continuous $\mathbb{R}$-valued functions $\phi$ defined on $[-\tau, 0]$ with the norm $\|\phi\|_{\tau}=\sup _{-\tau \leq \theta \leq 0}\|\phi(\theta)\|$, where $\tau$ is a positive constant.

$P C\left(J, \mathbb{R}^{n}\right)=\left\{\varphi: J \rightarrow \mathbb{R}^{n}\right.$ is continuous for all but at most a finite number of points $t \in J$ and at these points $t \in J, \varphi\left(t^{+}\right)$and $\varphi\left(t^{+}\right)$exists, $\left.\varphi\left(t^{+}\right)=\varphi(t)\right\}$, where $J \subset \mathbb{R}$ is a bounded interval, $\varphi\left(t^{+}\right)$and $\varphi\left(t^{+}\right)$denote the right-hand and left-hand limits of the function $\varphi(t)$, respectively. Especially, let $P C \triangleq P C([-\tau, 0], H)$.

Let $P C_{\mathrm{F}_{0}}^{b}([-\tau, 0], H)\left(P C_{\mathrm{F}_{t}}^{b}([-\tau, 0], H)\right)$ denotes the family of all bounded $\mathrm{F}_{0}\left(\mathrm{~F}_{t}\right)$-measurable, $P C([-\tau, 0], H)$-valued random variables $\phi$, satisfying $\|\phi\|_{\mathrm{L}_{2}}^{2}=$ $\sup _{-\tau \leq \theta \leq 0} \mathrm{E}\|\phi(\theta)\|_{H}^{2}$.

In this paper, we consider the following stochastic partial functional differential equation with impulses:

$$
\left\{\begin{array}{l}
d x(t)=[A x(t)+f(t, x(t-\tau))] d t+\sigma(t, x(t-\tau)) d \omega(t), t \geq 0, t \neq t_{k} \\
\triangle x\left(t_{k}\right)=x\left(t_{k}^{+}\right)-x\left(t_{k}^{-}\right)=I_{k}\left(x\left(t_{k}\right)\right), t=t_{k}, k \in \mathbb{Z} \\
x_{0}(s)=\phi(s) \in P C_{\mathrm{F}_{0}}^{b}([-\tau, 0], H), \quad s \in[-\tau, 0], \text { a.s. }
\end{array}\right.
$$

where $A: D(A) \subset H \rightarrow H$ is the infinitesimal generator of an analytic semigroup of linear operator $S(t)_{t \geq 0}$ on a Hilbert space $H$; $f: \mathbb{R}^{+} \times H \rightarrow H$ and $\sigma: \mathbb{R}^{+} \times H \rightarrow \mathcal{L}_{2}^{0}$ are jointly continuous functions; the fixed moment of time $t_{k}$ satisfies $0<t_{1}<t_{2}<\cdots<t_{k}<\cdots$, and $\lim _{k \rightarrow+\infty}=+\infty ; x\left(t_{k}^{+}\right)$and $x\left(t_{k}^{-}\right)$ 
represent the right and left limits of $x(t)$ at $t=t_{k}, k=1,2, \cdots$, respectively; $\triangle x\left(t_{k}\right)=x\left(t_{k}^{+}\right)-x\left(t_{k}^{-}\right)$represents the jump in the state $x$ at time $t_{k}$ with $I_{k}$ determining the size of the jump.

We also assume $0 \in \rho(-A)$, the resolvent set of $-A$. Then we know that there exist constant $M \geq 0, \gamma>0$ such that

$$
\|S(t)\| \leq M e^{-\gamma t}, \quad t \geq 0 .
$$

Definition 2.1 A stochastic process $x(t), t \in \mathbb{R}^{+}$, is called a mild solution of the system (1), if

(i) $x(t)$ is an $\mathrm{F}_{t}(t \geq 0)$ adapted process.

(ii) $x(t) \in H$ has a càdlàg path on $t \in \mathbb{R}^{+}$almost surely.

(iii) for arbitrary $t \in \mathbb{R}^{+}$, we have

$$
\begin{aligned}
x(t) & =S(t) \phi(0)+\int_{0}^{t} S(t-s) f(s, x(s-\tau)) d s+\int_{0}^{t} S(t-s) \sigma(s, x(s-\tau)) d \omega(s) \\
& +\sum_{0<t_{k}<t} S\left(t-t_{k}\right) I_{k}\left(x\left(t_{k}\right)\right)
\end{aligned}
$$

where $x_{0}(\cdot) \in P C_{\mathrm{F}_{0}}^{b}([-\tau, 0], H)$, a.s..

Definition 2.2 The mild solution of system (1) is said to be exponentially stable in mean square if there exists a pair of positive constants $\lambda>0$ and $M \geq$ 1 such that for any solution $x(t)$ with the initial condition $\phi \in P C_{\mathrm{F}_{0}}^{b}([-\tau, 0], H)$

$$
\mathrm{E}\|x(t)\|^{2} \leq M\|\phi\|_{\mathrm{L}_{2}}^{2} e^{-\lambda t}, t \geq 0 .
$$

\section{Main Results}

For system (1), we impose the following assumptions:

(A1) There exist constants $L_{f}>0, L_{\sigma}>0$ such that for any $x, y \in H$ and $t \geq 0$

$$
\begin{gathered}
\|f(t, x)-f(t, y)\| \leq L_{f}\|x-y\|, f(t, 0)=0, \\
\|\sigma(t, x)-\sigma(t, y)\|_{\mathcal{L}_{2}^{0}} \leq L_{\sigma}\|x-y\|, \sigma(t, 0)=0 .
\end{gathered}
$$

(A2) There exist some positive number $q_{k}(k=1,2, \cdots)$ such that

$$
\left\|I_{k}(x)-I_{k}(y)\right\| \leq q_{k}\|x-y\|, I_{k}(0)=0, k=1,2, \cdots,
$$

for any $x, y \in H$ and $\sum_{k=1}^{\infty} q_{k}<\infty$.

Under the assumptions:(A1)-(A2), the existence and uniqueness of mild solution to the system (1) is easily shown by using Picard iterative method. 
Theorem 3.1 Suppose the assumptions (A1)-(A2) hold, and we further assume that the following conditions

(A4) $\inf _{k=1,2, \ldots}\left(t_{k}-t_{k-1}\right)=\theta>0$,

(A5) $\left(3 M^{2} L_{f}^{2} \gamma^{-1}+3 M^{2} L_{\sigma}^{2}\right) e^{\gamma \tau}+\frac{1}{\theta} \ln \left(1+6 M^{2}\left(\sum_{k=1}^{\infty} q_{k}\right)^{2}\right)<\gamma$,

hold. Then the mild solution of system (1) is exponentially stable in mean square.

Proof.From (3), for any $t \geq 0$, we can get

$$
\begin{aligned}
& \mathrm{E}\|x(t)\| \\
= & \mathrm{E} \| S(t) \phi(0)+\int_{0}^{t} S(t-s) f(s, x(s-\tau)) d s+\int_{0}^{t} S(t-s) \sigma(s, x(s-\tau)) d \omega(s) \\
+ & \sum_{0<t_{k}<t} S\left(t-t_{k}\right) I_{k}\left(x\left(t_{k}\right)\right) \|^{2} \\
\leq & 6 \mathrm{E}\|S(t) \phi(0)\|^{2}+6 \mathrm{E}\left\|\sum_{0<t_{k}<t} S\left(t-t_{k}\right) I_{k}\left(x\left(t_{k}\right)\right)\right\|^{2}+3 \mathrm{E}\left\|\int_{0}^{t} S(t-s) f(s, x(s-\tau)) d s\right\|^{2} \\
+ & 3 \mathrm{E}\left\|\int_{0}^{t} S(t-s) \sigma(s, x(s-\tau)) d \omega(s)\right\|^{2} \\
= & \sum_{i=1}^{4} J_{i} .
\end{aligned}
$$

It follows from (2) that

$$
J_{1}=6 \mathrm{E}\|S(t) \phi(0)\|^{2} \leq 6\|S(t)\|^{2} \mathrm{E}\|\phi(0)\|^{2} \leq 6 M^{2} e^{-\gamma t}\|\phi\|_{\mathrm{L}_{2}}^{2}
$$

Combining (A2) with Hölder inequality, we can get

$$
\begin{aligned}
J_{2} & =6 \mathrm{E}\left\|\sum_{0<t_{k}<t} S\left(t-t_{k}\right) I_{k}\left(x\left(t_{k}\right)\right)\right\|^{2} \\
& \leq 6 M^{2} \mathrm{E}\left(\sum_{0<t_{k}<t} e^{-\gamma\left(t-t_{k}\right)} q_{k}\left\|x\left(t_{k}\right)\right\|\right)^{2} \\
& \leq 6 M^{2}\left(\sum_{k=1}^{\infty} q_{k}\right)\left(\sum_{0<t_{k}<t} e^{-2 \gamma\left(t-t_{k}\right)} q_{k} \mathrm{E}\left\|x\left(t_{k}\right)\right\|^{2}\right) \\
& \leq 6 M^{2}\left(\sum_{k=1}^{\infty} q_{k}\right)^{2}\left(\sum_{0<t_{k}<t} e^{-\gamma\left(t-t_{k}\right)} \mathrm{E}\left\|x\left(t_{k}\right)\right\|^{2}\right)
\end{aligned}
$$


From (A1) and Hölder inequality, we obtain

$$
\begin{aligned}
J_{3} & =3 \mathrm{E}\left\|\int_{0}^{t} S(t-s) f(s, x(s-\tau)) d s\right\|^{2} \\
& \leq 3 M^{2} L_{f}^{2} \mathrm{E}\left(\int_{0}^{t} e^{-\gamma(t-s)}\|x(s-\tau)\| d s\right)^{2} \\
& \leq 3 M^{2} L_{f}^{2}\left(\int_{0}^{t} e^{-\gamma(t-s)} d s\right)\left(\int_{0}^{t} e^{-\gamma(t-s)} \mathrm{E}\|x(s-\tau)\|^{2} d s\right) \\
& \leq 3 M^{2} L_{f}^{2} \gamma^{-1}\left[\gamma^{-1} e^{-\gamma t}\left(e^{\gamma \tau}-1\right)\|\phi\|_{\mathrm{L}_{2}}^{2}+e^{\gamma \tau} \int_{0}^{t-\tau} e^{-\gamma(t-s)} \mathrm{E}\|x(s)\|^{2} d s\right](7)
\end{aligned}
$$

Using (A1) and Burkholder-type inequality, we obtain

$$
\begin{aligned}
J_{4} & =3 \mathrm{E}\left\|\int_{0}^{t} S(t-s) \sigma(s, x(s-\tau)) d \omega(s)\right\|^{2} \\
& \leq 3 \mathrm{E} \int_{0}^{t}\|S(t-s)\|^{2}\|\sigma(s, x(s-\tau))\|_{\mathcal{L}_{2}^{0}}^{2} d s \\
& \leq 3 M^{2} L_{\sigma}^{2} \int_{0}^{t} e^{-2 \gamma(t-s)} \mathrm{E}\|x(s-\tau)\|^{2} d s \\
& \leq 3 M^{2} L_{\sigma}^{2}\left[\gamma^{-1} e^{-\gamma t}\left(e^{\gamma \tau}-1\right)\|\phi\|_{\mathrm{L}_{2}}^{2}+e^{\gamma \tau} \int_{0}^{t-\tau} e^{-\gamma(t-s)} \mathrm{E}\|x(s)\|^{2} d s\right]
\end{aligned}
$$

Substituting (4)-(9) into (3), we have

$$
\begin{aligned}
& \mathrm{E}\|x(t)\|^{2} e^{\gamma t} \\
\leq & 6 M^{2}\|\phi\|_{\mathrm{L}_{2}}^{2}+6 M^{2}\left(\sum_{k=1}^{\infty} q_{k}\right)^{2}\left(\sum_{0<t_{k}<t} \mathrm{E}\left\|x\left(t_{k}\right)\right\|^{2} e^{\gamma t_{k}}\right)+3 M^{2} L_{f}^{2} \gamma^{-2}\left(e^{\gamma \tau}-1\right)\|\phi\|_{\mathrm{L}_{2}}^{2} \\
+ & 3 M^{2} L_{f}^{2} \gamma^{-1} e^{\gamma \tau} \int_{0}^{t} \mathrm{E}\|x(s)\|^{2} e^{\gamma s} d s+3 M^{2} L_{\sigma}^{2} \gamma^{-1}\left(e^{\gamma \tau}-1\right)\|\phi\|_{\mathrm{L}_{2}}^{2} \\
+ & 3 M^{2} L_{\sigma}^{2} e^{\gamma \tau} \int_{0}^{t} \mathrm{E}\|x(s)\|^{2} e^{\gamma s} d s \\
= & C\|\phi\|_{\mathrm{L}_{2}}^{2}+p \int_{0}^{t} \mathrm{E}\|x(s)\|^{2} e^{\gamma s} d s+\beta \sum_{0<t_{k}<t} \mathrm{E}\left\|x\left(t_{k}\right)\right\|^{2} e^{\gamma t_{k}},
\end{aligned}
$$

where $C=6 M^{2}+3 M^{2}\left(L_{f}^{2} \gamma^{-2}+L_{\sigma}^{2} \gamma^{-1}\right)\left(e^{\gamma \tau}-1\right), p=3 M^{2}\left(L_{f}^{2} \gamma^{-1}+L_{\sigma}^{2}\right) e^{\gamma \tau}$, $\beta=6 M^{2}\left(\sum_{k=1}^{\infty} q_{k}\right)^{2}$.

According to Gronwall-Bellmen's Lemma ([1]), we have

$$
\mathrm{E}\|x(t)\|^{2} \leq C\|\phi\|_{\mathrm{L}_{2}}^{2} \prod_{0<t_{k}<t}(1+\beta) e^{-(\gamma-p) t} .
$$


On the other hand, by (A4), one has

$$
\prod_{0<t_{k}<t}(1+\beta) \leq(1+\beta)^{\frac{t}{\theta}}=e^{\frac{t}{\theta} \ln (1+\beta)} .
$$

Thereby, (10) can be rewritten as

$$
\mathrm{E}\|x(t)\|^{2} \leq C\|\phi\|_{\mathrm{L}_{2}}^{2} e^{-\left(\gamma-p-\frac{\ln (1+\beta)}{\theta}\right) t} .
$$

From the assumption (A5), it implies that the mild solution of system (1) is exponentially stable in mean square.

This completes the proof.

Theorem 3.2 Suppose that all the conditions of Theorem 3.1 hold. Then the mild solution of system (1) is exponential stable almost surely.

Proof.The proof is quite similar to the proof of Theorem 5.1 in [4], we omit it here.

If $I_{k}(\cdot) \equiv 0$, then system (1) becomes stochastic partial functional differential equations:

$$
\left\{\begin{array}{l}
d x(t)=[A x(t)+f(t, x(t-\tau))] d t+\sigma(t, x(t-\tau)) d \omega(t), t \geq 0 \\
x_{0}(s)=\phi(s) \in C_{\mathrm{F}_{0}}^{b}([-\tau, 0], H), \quad s \in[-\tau, 0], \text { a.s. }
\end{array}\right.
$$

Corollary 3.3 Assume (A1) holds and the following condition

$$
\left(3 M^{2} L_{f}^{2} \gamma^{-1}+3 M^{2} L_{\sigma}^{2}\right) e^{\gamma \tau}<\gamma
$$

holds. Then the mild solution of system (11) is exponentially stable in mean square.

Remark 3.4 In [9], Caraballo and Liu have studied the exponential stability in $p(p \geq 2)$-moment of mild solution to (11) by utlizing Gronwall inequality, the monotone decreasing behaviors of the delays are imposed. Particularly, when $\tau(t) \equiv \tau, \delta(t) \equiv \tau$, the condition ensuring the exponential stability in mean square is $\left(3 M^{2} L_{f}^{2} \gamma^{-1}+3 M^{2} L_{\sigma}^{2}\right) e^{\gamma \tau}<\gamma$. From Corollary 3.5, it is obvious that our results improve the results in [9].

ACKNOWLEDGEMENTS. The author would like to thank the referee and the editor for their careful comments and valuable suggestions on this work. This work is supported by Youth Foundation of Chongqing Three Gorges University (No.12QN24), Foundation for Professor and Doctor of Chongqing Three Gorges University (No.12ZZ36), Scientific Research Foundation of Chongqing Three Gorges University (No.12RC12). 


\section{References}

[1] A.M.Samoilenko and N.A.Perestyuk, Differential Equations with Impulsive Effect, World Scientific, Singapore, 1995.

[2] A.Pazy, Semigroup of Linear Operators and Applications to Partial Differential Equations, Springer-Verlag, New York, 1983.

[3] D.D.Bainov and P.S.Simeonov, Theory of Impulsive Differential Equations: Periodic Solutions and Applications. Longman, Harlow, 1993.

[4] J.W.Luo, Stability of stochastic partial differential equations with infinite delays, J.Comput.Appl.Math., 222 (2008), 364 - 371.

[5] J.W.Luo, Fixed point and exponential stability of mild solutions of stochastic partial differential equation with delays, J.Math.Anal.Appl., 342 (2008), 753 - 760.

[6] K.Liu and Y.Shi, Razuminkhin-type theorems of infinite dimensional stochastic functional differential equations, In:IFIP, System, Control, Modeling and Optimization, (2006), 237 - 247.

[7] R.Sakthivel and J.W.Luo, Asymptotic stability of impulsive stochastic partial differential equations with infinite delays, J.math.Anal.Appl., 356 (2009), 1 - 6 .

[8] R.Sakthivel, J.W.Luo, Asymptotic stability of impulsive stochastic partial differential equations, Statist.Probab.Lett., 79 (2009), 1219 - 1223.

[9] T.Caraballo and K.Liu, Exponential stability of mild solutions of stochastic partial differential equations with delays, Stoch.Anal.Appl., 17 (1999), $27-47$.

[10] T.Taniguchi, Asymptotic stability theorems of semilinear stochastic evolution equations in Hilbert space, Stoch.Stoch.Rep., 53 (1995), 41 - 52.

[11] T.Taniguchi, Almost sure exponential stability for stochastic partial functional differential equations, Stoch.Anal.Appl., 16 (1998), 965 - 975.

[12] T.Taniguchi, K.Liu and A.Truman, Existence, uniqueness and asymptotic behavior of mild solutions to stochastic functional differential equations in Hilbert space, J.Differential Equations, 181 (2002), 72 - 91.

[13] T.Taniguchi, The exponential stability for stochastic delay partial differential equations, J.Math.Anal.Appl., 331 (2007), 191 - 205.

\section{Received: November, 2012}

